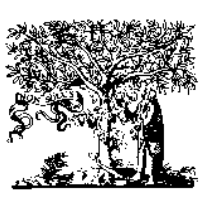

ELSEVIER

\title{
Evaluating the reliability of prototype degradable systems
}

\author{
M.L. Leuschen ${ }^{\mathrm{a}, *}$, I.D. Walker ${ }^{\mathrm{b}}$, J.R. Cavallaro ${ }^{\mathrm{a}}$ \\ ${ }^{\mathrm{a}}$ Department of Electrical and Computer Engineering, Rice University, Houston, TX 77005, USA \\ ${ }^{\mathrm{b}}$ Department of Electrical and Computer Engineering, Clemson University, Clemson, SC 29634, USA
}

Received 1 February 2000; accepted 2 September 2000

\begin{abstract}
The technique introduced in this paper is a new technique for analyzing fault tolerant designs under considerable uncertainty, such as seen in unique or few-of-a-kind devices in poorly known environments or pre-prototype design analyses. This technique is able to provide useful information while maintaining the uncertainty inherent in the original specifications. The technique introduced here is a logical extension of the underlying concepts of fuzzy sets and Markov models. Although originally developed for robotic systems, the technique is more broadly applicable. This paper develops fuzzy Markov modeling and uses it to analyze a specific robot designed for hazardous waste removal and specific types of electronic systems. (C) 2001 Elsevier Science Ltd. All rights reserved.
\end{abstract}

Keywords: Fault trees; Markov modeling; Robotics; Interval arithmetic; Fuzzy sets

\section{Introduction}

There has recently been significant work in the development and analysis of fault tolerant robots [1-3]. Much of this work has centered on incorporation of redundant [4-6] and backup systems. The extra components needed for fault tolerant robot designs obviously add extra costs and extra possibilities of failure [7,8]. Reliability analysis tools such as fault trees [9-11] and Markov [12-14] models give hard numbers showing that the benefits of the fault tolerant design are tangible and worth the effort. Unfortunately, the typical robotic component failure rates required for these calculations are generally very dependent on configuration and environment and thus known only approximately during the design phase [10]. A method of considering the full range of possible failure rates is needed to give a good idea of what is and is not known.

The standard approaches of reliability engineering rely wholly on the probability model, which is often inappropriate for this task $[10,15,16]$. Probability based analyses usually require more information about the system than is known, such as mean failure rates, or failure rate distributions. Commonly, this results in dubious assumptions about the original data. As a result, any single value or distribution used to describe poorly known failure characteristics is likely to give a result that is misleading.

\footnotetext{
* Corresponding author. Fax: +1-713-348-6196.

E-mail address: martinl@ rice.edu (M.L. Leuschen).
}

For example, consider a system design where there are two independent modes of failure. Due to the complexities of the system, a hostile work environment, and lack of a prototype, the failure rates of these modes are only known to the nearest order of magnitude. (This is common in reliability databases [17].) Analysis using the means of the expected failure rates implies that the two failures are equally likely, while in fact it is possible that one type of failure will be 100 times more likely than the other. A more complex analysis that maintained the uncertainty of the system might note this possibility, but would state that it was very unlikely. This is not necessarily true - it is an artifact of the assumption that the failure rate has some arbitrary (often Gaussian) distribution.

A strong argument for fuzzy reliability analysis can be found in Ref. [16] where it is demonstrated that a fuzzy reliability analysis of the Chernobyl reactor would have revealed the unreliability that caused the disaster there. Fuzzy logic offers an alternative to the probability paradigm, possibility, that is much more appropriate to reliability in contexts where failure characteristics are poorly known, such as prototyping and robotics $[10,11,15,16]$. Some researchers advocate the method of coherent imprecise probabilities [18] as opposed to the interval possibilities used in this paper. However, the approach taken in this paper is to adopt the more familiar and intuitive possibility model. Possibility mathematics allows for quantitative reliability calculations that preserve the uncertainty present in the original data. The possibility model deals 


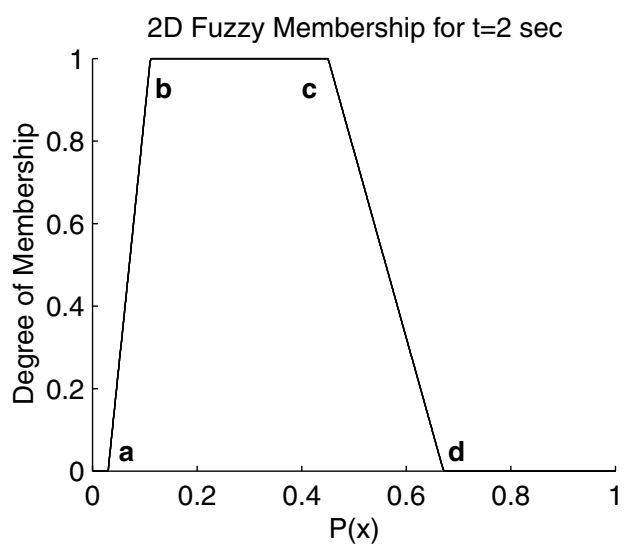

Fig. 1. A fuzzy membership function.

with uncertainty in a way that avoids making unwarranted assumptions, and makes the consequences of the required assumptions clear.

Of the common reliability tools, only fault trees, event trees, and similar variants have been fuzzified to any great extent. However, while fault trees are very useful, they are somewhat limited in their applications. Partial failures, coverage, repairable systems, and other important reliability issues are not covered well by the fault tree approach, although recent developments in fault tree analysis are expanding the range of application somewhat $[19,20]$. Markov modeling is a valuable tool for dealing with the above situations, and indeed, is often used in the special cases mentioned above. However, there has been little previous work on fuzzy Markov modeling. It will be shown in the following section that this previous work is inappropriate for reliability applications. In Section 3, we introduce a new method of fuzzy Markov modeling that is suitable for reliability engineering. This is illustrated by examples including a prototype hazardous waste cleanup manipulator in Section 4 and a typical RAID disk drive system in Section 5.

\section{Fuzzy Markov models}

In this section we review fuzzy Markov methods, and discuss the limitations of the current fuzzy Markov techniques for reliability engineering. The classical reliability Markov model divides the possible configurations of the system into a number of states. Each of these states can be connected to some of the other states by a crisp transition rate, a fixed probability of instantaneously entering the other state. The probability of being in each state (or population of that state) evolves over time according to these rates. Knowledge of the current state provides all the information known about the condition of the model system. Explicit information about the past states and transitions of the system is thus not available. The system is said to be memoryless, or to have the Markov property. For reliability Markov models, the probabilistic transitions usually represent part failures. These models are most valid when failure probabilities are indeed roughly constant over time, and most useful to examine the behavior of a system where the order of failure events over time is important.

A significant problem with Markov models is that we often do not know the failure rate probabilities very accurately. An order of magnitude of uncertainty is not at all unusual. A common method of dealing with this is to estimate a single non-fuzzy, or crisp, probability and assume it is sufficient. A more sophisticated approach would be to assign a probability distribution to each of these probabilities, resulting in probabilities of probabilities. As discussed in the previous section, these assumptions are often inappropriate.

For the new fuzzy Markov models introduced in this paper, both the state populations and the transition rates will be fuzzy. Existing fuzzy Markov models have crisp populations, which imply unwarranted certainty about the probability of being in a state. The transition rates must be fuzzy because we only have fuzzy data on their values. The populations change with time according to these fuzzy transition rates, and thus must become fuzzy themselves. Proper
3D Fuzzy State Plot

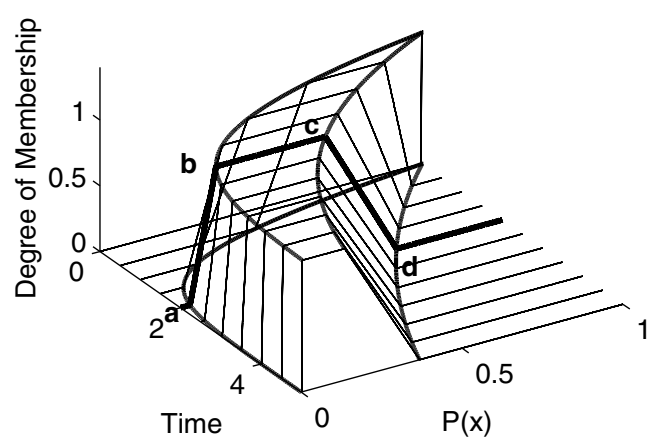

3D Fuzzy State Plot

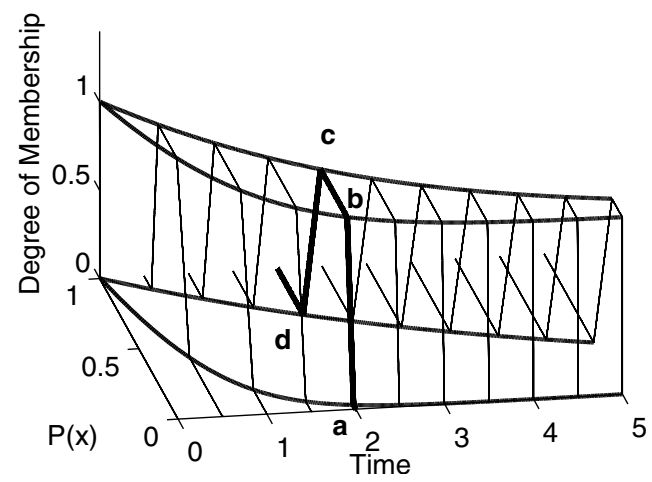

Fig. 2. Fuzzy output over time in 3D, two views. 


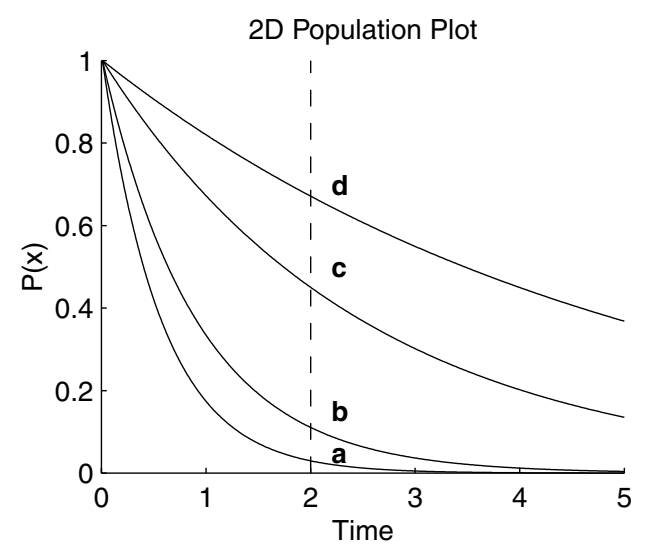

Fig. 3. Fuzzy output over time in 2D.

mathematics to describe the interaction between the two will be required to produce a valid fuzzy Markov model.

The first problem is to decide on a format for the fuzzy sets to be used in the model. Our approach is to estimate the conservative and optimistic bounds of the transition probabilities in question, and use them to define a trapezoidal membership function. This estimate is reasonably easy to perform for most systems, and has the benefit of being clear cut and easy to understand and modify. We will use the conservative bounds for the base, and the optimistic bounds for the top, as seen in Fig. 1. These bounds are referred to as the zero-cut and one-cut, respectively, as they mark the sets where the membership function is greater than or equal to zero and one. (In general, an $\alpha$-cut is the set where the membership function is greater than or equal to $\alpha$ [21].) The fuzzy failure rate or population may be listed as a four-element vector, with the one-cut surrounded by the zero-cut. For example, if the optimistic bounds of the state population are $[0.10,0.45]$ and the pessimistic bounds are $[0.04,0.65]$, the population may be represented as $[0.04,0.10,0.45,0.65]$. Fig. 1 shows the membership function corresponding to this vector.

The output for our fuzzy Markov model is by necessity three dimensional, since we need to represent all three dimensions of probability, degree of membership (possibility), and time. However, if we only plot the corners of the trapezoidal possibility distributions, this can be reduced to two dimensions without losing any information. This can be seen in Figs. 1-3, where we show several views of the exponentially decaying state population of a simple system.
(To look ahead, this result represents the initial state IN from Fig. 4, with the state population at $t=0$ equal to $[1,1,1,1]$.) To aid comprehension, the fuzzy membership function for $t=2 \mathrm{~s}$ has been highlighted, and the corners labeled, on all figures.

Once the desired format of the fuzzy Markov model was determined, it became necessary to establish a formal mathematical method using this format. The proper approach to dealing with fuzzy populations in the Markov context is not intuitively obvious. Several useful criteria are established below. These will allow us to easily describe the validity and utility of various methods of fuzzification proposed in later sections.

The first criterion is simple mathematical accuracy. A valid fuzzy model will properly process both probabilities and fuzzy uncertainties. This is important as some existing fuzzy applications [22,23] preserve less fuzzy information than required in the model proposed above. Since its purpose is to accurately portray the uncertainties inherent in prototyping, existing fuzzy methods that rely on simplification will be inappropriate. This requirement will be referred to as the uncertainty criterion.

Another important factor is complexity. The fuzzy Markov model uses fuzzy possibility densities where the crisp model uses single crisp probabilities. A crisp model can be represented by sets of first-order differential equations, and solved using standard linear algebra techniques. For the fuzzy Markov model described above, both the constants and the variables in these equations have been replaced with fuzzy membership functions. Although an effort has been made to reduce complexity by using simple trapezoidal membership functions, this substitution complicates the computation considerably. As it is important for a practical reliability model to be reasonably easy to use, this is an important issue. The desire for such a model will be referred to as the complexity criterion.

Another type of criterion that we will judge our new fuzzy Markov Model on will be the kind of data it generates. Investigation showed that many standard fuzzification methods could result in mathematically dubious data. We define two more criteria, fuzzy validity and probabilistic validity, to deal with the two most common problems. For our purposes, any piecewise continuous function bounded on the $[0,1]$ interval is a valid fuzzy membership function [13]. The requirement for probabilistic validity is that we do not ever have any
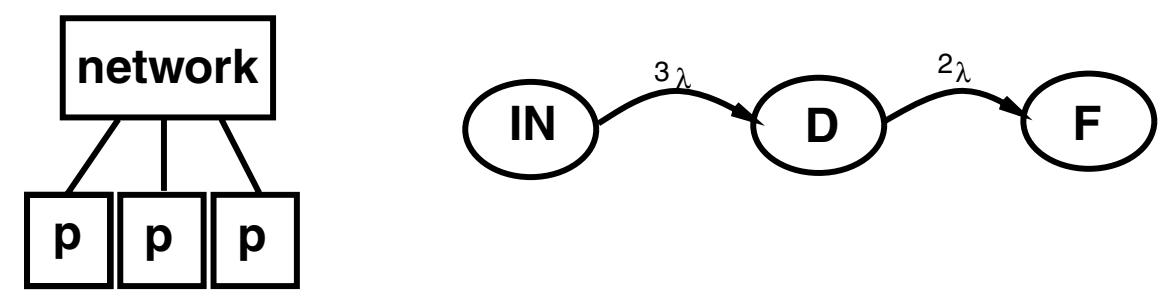

Fig. 4. Parallel processor system. 
possibility greater than zero of probabilities outside of the $[0,1]$ interval. Normally probability theory ensures this, but this is not necessarily compatible with the fuzzy mathematics needed to ensure the first validity criterion. For example, the probabilistic axiom 'the sum of all probabilities equals crisp one' is impossible to enforce under fuzzy mathematics. For fuzzy numbers, this can only be true in the sense of fuzzy one.

\subsection{Inappropriate fuzzy Markov models}

In this section we briefly discuss several unsuccessful methods we investigated for solving a fuzzy reliability Markov model. These methods are of interest because they show how established fuzzy reliability approaches from other areas fail to solve the problem, while giving some insight into the mathematical structure of our model. Although space constraints limit the detail presented in this paper, a more complete discussion is available in Refs. $[13,24]$.

We initially investigated methods similar to those used for fuzzy fault trees $[10,11,21]$. For these it can be sufficient to propagate the various corresponding $\alpha$-cut end points through the fault tree as if they were crisp, and then take the resulting extremal points as the corners of the output possibility distribution $[10,13,14,21]$. Unfortunately, this method requires many constraints inherent in the fault tree method which are not present for Markov models, and thus violates the uncertainty criterion in all but the most trivial Markov systems. Fuzzy fault trees themselves are fuzzy mathematically accurate, but the method does not extend to Markov models.

A basic technique for the extension of a crisp binary operation to a fuzzy operation is the extension principle. It can be defined as follows [21,25]:

Let $u(a)$ and $v(b)$ be fuzzy membership functions defined over crisp sets $A$ and $B$ and let $f(a, b)=y$ be a crisp binary operation. (Thus $y$ is crisp as well.) The equivalent fuzzy operation $f(u(a), v(b))=\mu(y)$ maps membership functions $u$ and $v$ to membership function $\mu$. Then the fuzzy result is $\mu(y)=f(u, v)=\sup (\min (u(a), v(b)))$ over $a, b$ S.T. $f(a, b)=y$.

The operation of a Markov model can be expressed in terms of binary operators, so the extension principle is a logical tool to apply. However, we determined that this approach violated the probabilistic validity criterion: probabilities outside the $[0,1]$ interval are considered possible by this model. This is a result of the way fuzzy arithmetic violates the axioms of probability, as discussed above.

Previous work also exists discussing fuzzy Markov modeling through a technique called fuzzy integration [23]. Unfortunately, fuzzy integrals are inappropriate for the reliability application. Although a fuzzy integral does determine the possibility of a fuzzy event, the result of such an integral is a single fuzzy value for a constant rather than a fuzzy membership function over a set [23]. Although this is a logical approach in some instances, we wish to propagate the uncertainty described by the fuzzy membership functions intact through our reliability models. Since they do not propagate uncertainty, fuzzy integral Markov models violate the uncertainty criterion, and not useful in our context.

\section{New fuzzy Markov model approach suitable for reliability}

As we have seen in Section 2, previously developed fuzzy Markov models are inappropriate for reliability analysis. In this section, we investigate an alternative methodology and discuss its features and strengths. Earlier in this paper, we decided to use the approach where we only considered the extremal values of the trapezoidal membership function. It has been shown that this approach simplifies the complexities of the fuzzy set into two easy to understand intervals, without much loss of information $[10,11,21]$. Once we have done this, interval mathematics gives us a simple rule on how to extend binary operations to intervals. The basic definition of interval extension is [26]:

$A \circ B=\bigcup_{(a \in A, b \in B)}(a \circ b)$

where $A$ and $B$ are arbitrary independent intervals and $\circ$ is an arbitrary binary operation. This is the definition of extension from first principles. It states that the result of an interval binary operation is the union of the original binary operation on all possible combinations of elements in those intervals.

Note that if $A$ and $B$ are not independent, the definition above must be modified. A common issue for reliability applications is when $A$ and $B$ represent the same thing this is called the 'repeated variable problem' [27]. In the method developed below, this is dealt with by assigning all instances of the same variable the same value in individual runs. Otherwise, the standard simplifying assumption that variables are independent is used.

This definition can be used on the intervals describing the trapezoidal membership functions used in our fuzzy Markov model. Simply solve the Markov model for the possible combinations of elements and take the union of the results. The fuzzy calculations are replaced by crisp calculations and elementary set theory, and the result is demonstrably correct by first principles.

Unfortunately, this approach has its own problems. Since an interval contains an infinite number of points, one in theory needs an infinite number of Markov models to solve the problem. Although this is clearly impossible, if one assumes some smoothness, one can reduce this to a close sampling of these values instead of a continuum. (Indeed, this method will be referred to as the close sampling method from now on.) Areas on the population 


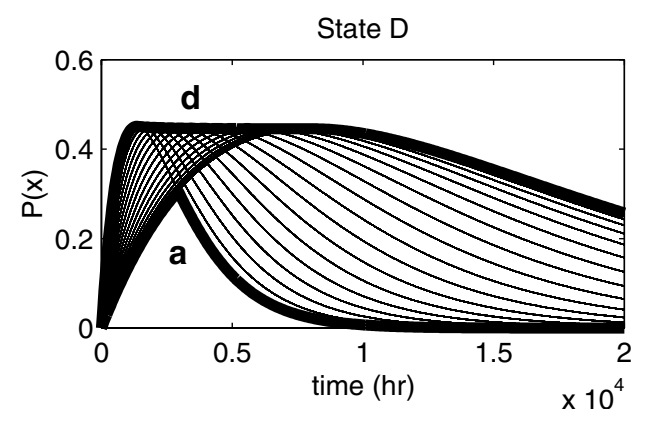

Fig. 5. Fuzzy Markov modeling of state D through close sampling method.

graph that are between different plots are covered by some probability value between the values that resulted in those plots. Complexity for this approach is high, but a solution to the problem is now possible.

Consider the fault tolerant parallel processor system shown in Fig. 4, a subset of a larger system discussed in Ref. [28]. This system uses three processors in a voting scheme but requires only two processors for functionality. Thus, a Markov model of the system has three states: IN, D and F. These represent the initial, undamaged state, the damaged state with only two working processors, and the failed state, respectively. Processors fail at rate $\lambda$, equal to 0.0011 per hour [28].

However, as discussed previously, we do not always have definite knowledge of actual failure rates, especially in the system prototyping phase. In Fig. 5, we use the close sampling method to calculate the conservative bounds represented by "a" and "d" in our trapezoidal representation of a fuzzy processor failure rate equal to [0.0005,0.003]. Twenty-one crisp Markov models are used to determine the fuzzy model over a period of 20,000 h. The thin curves represent the crisp Markov models determined by different (geometrically spaced) samples of the set of fuzzy failure rates. The thick bounding lines "a" and "d" represent the output of the model - the upper and lower feet of the trapezoidal fuzzy set as seen in Fig. 5. Note that for this simple system, the Markov model state $D$ is easy to calculate: $D(t)=3 \mathrm{e}^{-2 \lambda t}-3 \mathrm{e}^{-3 \lambda t}$.

Due to its more complete consideration of the structure of the situation, this approach meets all of our requirements listed for the fuzzy Markov model except for complexity. With sufficiently close sampling intervals, the uncertainty in our data is transmitted accurately, as the method follows first principles. Similarly, the smooth curves drawn along extremes of the crisp plots are themselves not overly complex and are easy to interpret.

The remaining fuzzy Markov models in this paper are calculated using the close sampling method above. Several samples were taken over each interval, spaced in a geometric fashion. (This concentrates samples near the lower values and gives smoother output curves.) The lower edge of the interval was the first sample, the upper the last. The resulting crisp Markov models were solved

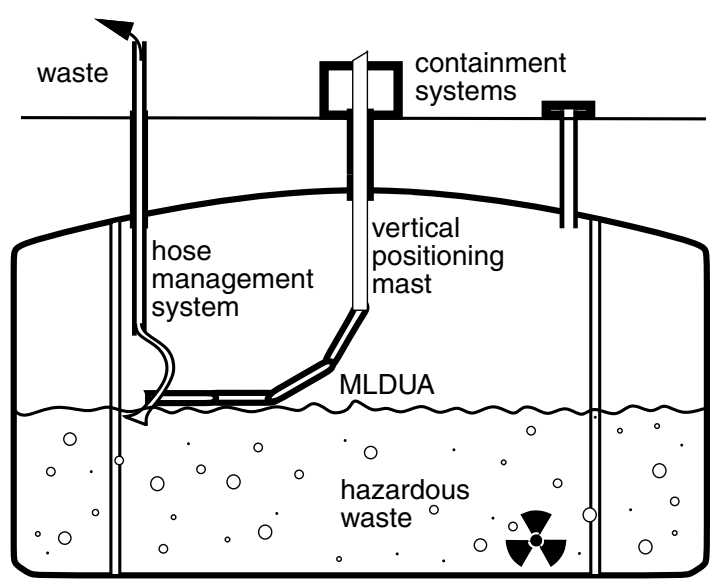

Fig. 6. MLDUA manipulator in waste tank.

using a finite-difference method [13], but any accurate Markov modeling method should give virtually identical results. Note that the symbolic solution to the Markov model can be especially useful in this case, as it can significantly reduce computation. (This is discussed further below.)

Note however, close sampling requires that many crisp Markov models be solved to solve a single fuzzy Markov model. If one is taking $N$ samples on the interval, and there are $M$ fuzzy failure rates, $N \times M$ crisp Markov models must be solved. As $N$ is in our applications typically on the order of 5-20, this can quickly grow to a significant number of calculations.

This close sampling approach appears to be the formulation best suited to calculating fuzzy Markov models for reliability applications and calculations. Despite the complexity issue, it is the only method that neither loses the important uncertainty information nor results in impossible or useless output. Thus, the original problem of finding a fuzzy Markov model becomes the problem of simplifying and implementing the close sampling fuzzy Markov model.

\subsection{Simplifying the Markov model for use with close sampling}

In systems with many similar components in similar roles, simplification can be accomplished by grouping the

Table 1

MLDUA component failure rates per $1000 \mathrm{~h}$

\begin{tabular}{llll}
\hline Component & Failure rate & Component & Failure rate \\
\hline Bearing & 0.00291 & Power supply & 0.0137 \\
Electric motor & 0.0092 & Rotary joint & 0.0075 \\
Electronic timer & 0.0012 & Sensor, general & 0.00361 \\
Hydraulic motor & 0.540 & Sensor, level, liquid & 0.0026 \\
Hydraulic pump & 0.0470 & Sensor, pressure & 0.00923 \\
Hydraulic valve & 0.00882 & Sensor, temperature & 0.00182 \\
Mechanical brake & 0.1386 & Strainer (filter) & 0.00019 \\
Optical encoder & 0.0155 & - & - \\
\hline
\end{tabular}


Table 2

MLDUA fuzzy system failures per $1000 \mathrm{~h} \alpha$-cuts

\begin{tabular}{lll}
\hline System failure & Zero-cut & One-cut \\
\hline Power & {$[0.0038,0.7284]$} & {$[0.0105,0.2755]$} \\
Joint & {$[0.2249,33.0053]$} & {$[0.6184,12.5873]$} \\
Servo control & {$[0.0000,0.00002]$} & {$[0.0000,0.0000]$} \\
Brake & {$[0.0139,2.0689]$} & {$[0.0383,0.7825]$} \\
Limping & {$[0.0113,1.6799]$} & {$[0.0311,0.6356]$} \\
Joint (damaged) & {$[0.1807,36.3309]$} & {$[0.4967,20.0215]$} \\
Servo control (damaged) & {$[0.0000,0.00002]$} & {$[0.0000,0.0000]$} \\
\hline
\end{tabular}

failures of these components together in the Markov model. Instead of having a state representing 'pressure sensor 23 has failed', for example, we have 'a pressure sensor has failed'. Provided the failure of any single sensor has a similar effect on the system, this is a valid simplification, and commonly used to simplify standard Markov models. This often also allows the use of a single possibility distribution for all of the similar components, reducing the number of crisp Markov models that must be solved considerably. This technique is referred to as component grouping. A complex system with many different parts will often have many fuzzy failure rates to deal with. Sometimes this is enough to make a fuzzy Markov model impractical even after component grouping. However, when examining the failure characteristics of any complex system, it is often useful to organize it into subsystems. This increases our understanding of the system by allowing us to focus on smaller, more comprehensible chunks. For example, if we were examining the failure characteristics of a robot arm, we might want to consider joint failures in our primary analysis. Once we knew those characteristics, we could then sharpen our focus to a model of the individual joints, considering motor, sensor, and mechanical failures, and so forth. This type of hierarchical simplification comes naturally and is helpful in promoting greater understanding of the system.

We can use this natural scheme of organization to simplify our fuzzy Markov models. Fuzzy fault trees are ideal for this purpose. They are easy to implement, fuzzy mathematically sound, and specifically designed to determine failure rates for collections of components. For each subsystem, we can implement a fuzzy fault tree as seen in Ref. [10] and use it to determine an accurate net fuzzy failure rate for the entire system.

Between component grouping and fuzzy fault trees, we have been able to reduce the number of fuzzy failure rates in a system to a manageable number. We can then sample this reduced set of failure rates and implement a fuzzy Markov model to examine the higher-level behaviors of the system without getting excessive complexity. Correct implementation will allow us the analysis benefits of both fuzzy mathematics and Markov modeling by using existing tools to deal with the parts of the system that do not require these computationally expensive techniques.

\subsection{Fuzzy Markov modeling algorithm}

The following is a summary of the fuzzy Markov modeling method:

1. Create a classic Markov model of the system of interest. Keep the number of distinct failure rates in the system low via component grouping and fuzzy fault trees.

2. Determine the appropriate fuzzy trapezoidal membership functions for the failure rates and initial populations.

3. Select crisp samples of the members of the one-cut and zero-cut sets representing the fuzzy membership functions.

4. Solve the Markov model for all the combinations of the samples and save the results.

5. Compute the maximum and minimum of the set of results corresponding to the one-cut and zero-cut of the model parameters, as seen in Fig. 5.

Fuzzy Markov modeling using this algorithm shows much promise as a reliability tool, as demonstrated by the following examples.

\section{Failed States}

L Limping Valve

J Joint Failure

F MLDUA Failure,

T MLDUA Trapped

Failure Rates

Joint plus Servo: js

Brake plus Power: pb

Limping Valve: 1

Damaged System: jo

Abort Rate for Damaged System: c

(Conservative operator only)
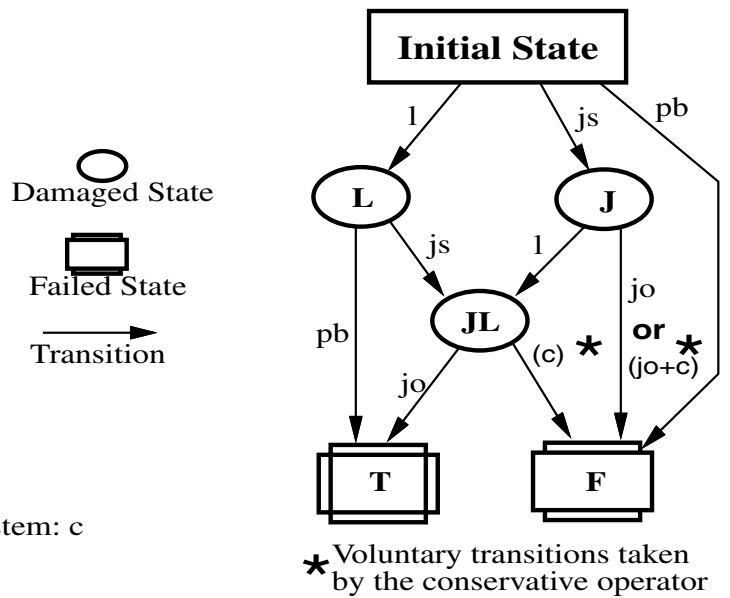

Fig. 7. MLDUA manipulator Markov model. 

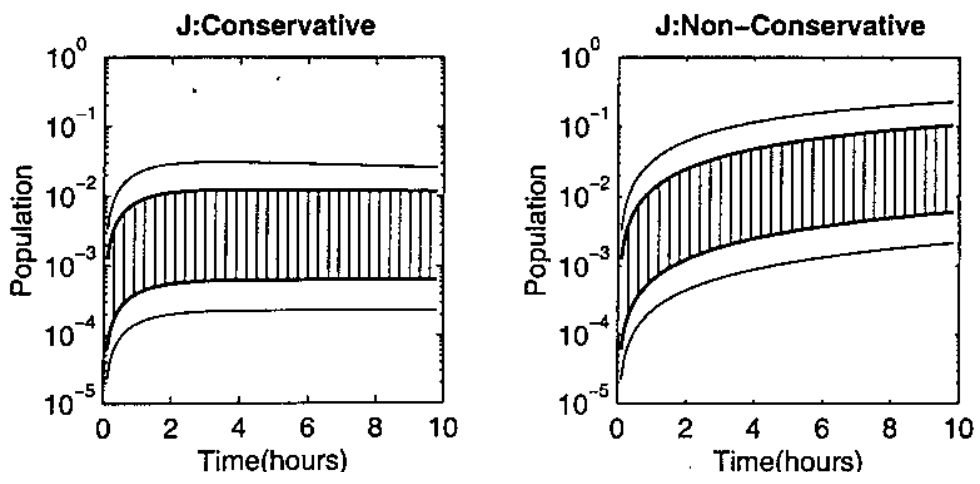

Fig. 8. MLDUA manipulator Markov model output for joint damaged state (J).

\section{Fuzzy Markov modeling of the MLDUA robot system}

The Modified Light Duty Utility Arm (MLDUA) is a robot arm designed to assist in the removal of hazardous radioactive waste from large underground storage tanks at Oak Ridge National Laboratory [9,29]. The MLDUA is inserted through a narrow central access riser, and used to manipulate a 'hose management system' for waste extraction, as seen in Fig. 6.

The environment in these tanks is extremely hazardous, and the waste involved is too dangerous to allow leakage. The MLDUA system has to meet many stringent safety requirements to deal with this [9]. In addition, the environment inside the tank is so hostile that the MLDUA itself is endangered. Extremely high radiation levels combine with explosive and corrosive chemicals to make the tank environment extremely dangerous to the robot. The overall effect of this environment on the robot cannot be predicted accurately before deployment. Stringent reliability requirements and uncertain failure characteristics thus combine to make the MLDUA system an ideal real world test case for fuzzy reliability analysis.

Considerable reliability work has already been done for the MLDUA. The design itself is very reliability conscious. Each joint is monitored by two sensors. Five of the seven joints are powered by hydraulic motors connected to a 'limping system', which will allow the robot to be straightened out and removed from the tank without power. This is an important consideration, as the robot arm is inserted through a narrow riser and must be 'limp' (straight) to remove from the tank. Due to the hazardousness of the tank's contents, only severely limited options are available for in-tank repair if the system fails [13].

An extensive fault tree analysis of the MLDUA system has been conducted previously [9]. This analysis considers the overall failure of the MLDUA system as well as tracking numerous lesser failures as subsidiary events. The events of interest are component failures that lead to failure of the MLDUA while operating in the tank. Power system failure, joint failure, braking system failure, servo control failure, and limping system failure are all considered as separate events modeled by trees, as found in Ref. [9]. In the following we apply the technique of fuzzy Markov modeling using fuzzy fault trees introduced in Section 3 to the analysis of the MLDUA.

Table 1 gives the mean failure rates, in failures per thousand hours of operation found in Ref. [17] for the typical components that may be used in this robot. These are then fuzzified as appropriate $[10,11,13]$ before use in the fault tree. This is a simple proportional operation where the one-cut and zero-cut are determined from the 68 and 90\% confidence intervals given in Ref. [17] corresponding to $[0.22,4.5]$ and $[0.08,11.9]$ times the nominal failure rate, respectively. Also, the frequencies of several events, such as pressure errors in the hydraulic system were not known at all. For these, a fuzzy representation of 'unknown', with
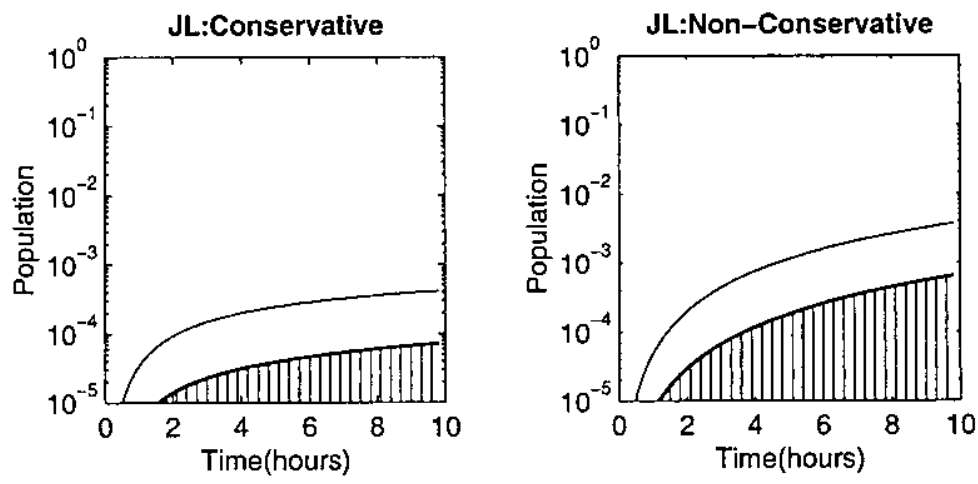

Fig. 9. MLDUA manipulator Markov model output for joint and limping system damaged state (JL). 

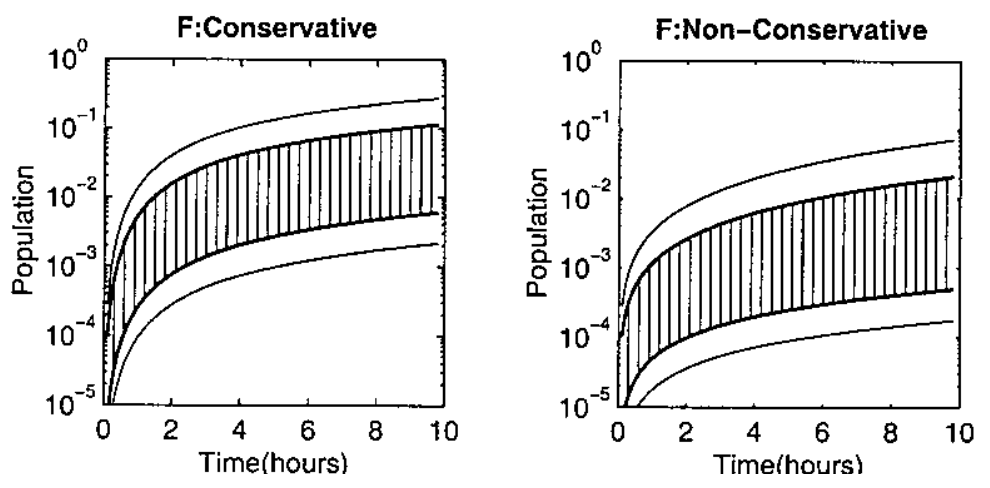

Fig. 10. MLDUA manipulator Markov model output for failed state (F).

both $\alpha$-cuts $=[0,1]$ is used. (Note that fuzzy Markov models can deal with completely unknown quantities like this without modification!)

Fuzzy Markov modeling of the MLDUA system is of interest to us due to the importance of the order of occurrence of a number of the system failures. Two cases are considered, based on different operational scenarios. In the first, a non-conservative optimistic operator runs the MLDUA for up to $10 \mathrm{~h}$ at a time, stopping only if total system failure occurs. The second case considers a conservative operator who removes the MLDUA shortly after any joint failure, in order to avoid a subsequent total failure combined with a limping failure, which may result in a trapped robot. Between uses, the strict maintenance schedule of the robot is expected to return the system to a working condition. The failure rates for both situations are calculated using fuzzy fault trees [13] as shown in Table 2. The possibly high failure rates indicated must be taken seriously due to the hazardous environment the MLDUA operates in.

Fig. 7 shows the Markov model used for both of these cases. The $\mathbf{J}$ state, representing a single failed joint, shows the redundancy in the manipulator. If one joint fails, the robot can continue to work at somewhat decreased efficiency. The L state represents the failure of the limping mechanism. The limping mechanism is not easily instrumented, so the operator is not able to distinguish between the Initial and L, or between the J and JL states. The conser- vative operator tries to make up for this lack and avoid trapped (state T) failures by voluntarily leaving the tank (effectively placing the robot in failed state F) relatively soon (transition rate $c$ is $1 \mathrm{~h}$ ) after any serious failure is noted.

In Figs. 8 and 9 we see the results of the fuzzy Markov model for the states J and JL. In these figures the one-cut is shaded to emphasize the region of highest possibility. (This region is equivalent to the area between the " $b$ " and " $c$ " curves in Figs. 1-3.) Note that the lower bounds of the fuzzy populations for state JL are off scale in Fig. 9.

In both cases we see that the conservative operator is less likely to be in these states, due to the doctrine of leaving the tank (and thus entering state F) as soon as a fault is detected. Both operators average the same number of faults, but the conservative one quits out of the $\mathbf{J}$ states as soon as possible, reducing the chance of being in these states at any given time. As the robot is still useful in these states, the conservative operator gets less work done overall.

Fig. 10 shows the results for the failed state (state F), representing the failure of the robot to complete its task and subsequent successful removal from the tank. It is evident that there is a high possibility that the MLDUA will not survive through the average working day without a work halting failure. This is not encouraging, but it is not surprising either, considering the complex nature of the system and hostile environment. Careful daily maintenance should reduce this problem. The price of conservative
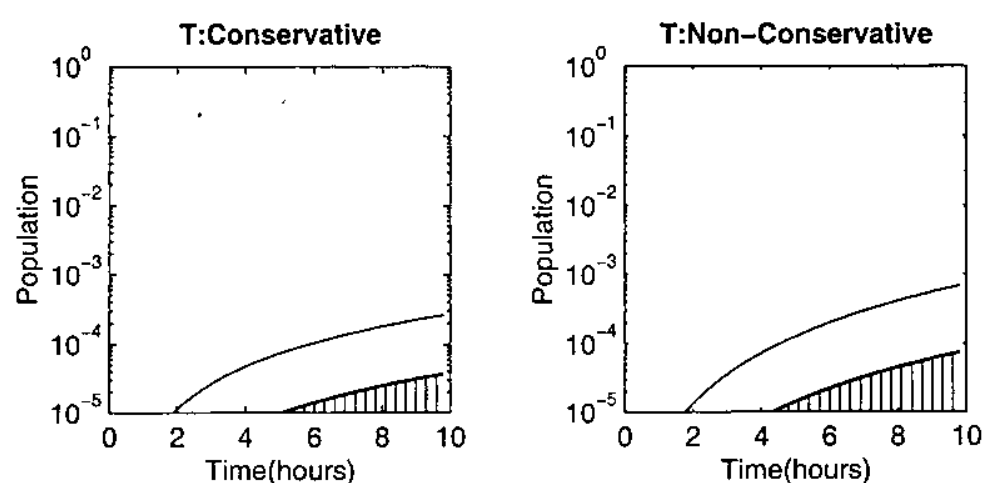

Fig. 11. MLDUA manipulator Markov model output for trapped state (T). 
T:Ratio

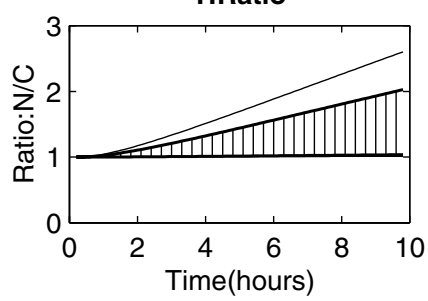

Fig. 12. Ratio of non-conservative to conservative operators, trapped state.

operation is also very obvious here, increasing worst-case failure probabilities by about half of an order of magnitude by the end of a working day.

Fig. 11 shows the fuzzy Markov model output for the trapped state. One can note that the possible probabilities for the 'trapped' state (state T) are fairly low for both Markov models, with worst-case values of less than one in a thousand. This may or may not be an acceptable risk level, depending on expected frequency of use and on the effectiveness of contingency plans for dealing with this failure. It motivates possible future investigation of fault tolerance and detection, as well as efficient reconfiguration, of the MLDUA.

State $\mathrm{T}$ is where conservative operation pays off, decreasing the chance of being trapped (state $\mathrm{T}$ ) by up to three times in the worst-case scenario, as seen in Fig. 12. (In the best case, this failure hardly happens at all, so there is little benefit from avoiding it.) It is worth considering if this worst-case reduction is worth the associated reduction in work accomplished. A cost-benefit analysis would be in order, following the results of the fuzzy Markov modeling here, to facilitate a higher-level decision about operational doctrine.

Here one of the advantages of the fuzzy Markov model becomes evident. This decision might not be made by a reliability engineer. The fuzzy nature of the results make the uncertainty in any such analysis very clear to anyone with even a nodding acquaintance with fuzzy mathematics, regardless of their understanding of the system or reliability theory in general. The uncertainty is built in to the results. Similarly, this uncertainty is mathematically determined

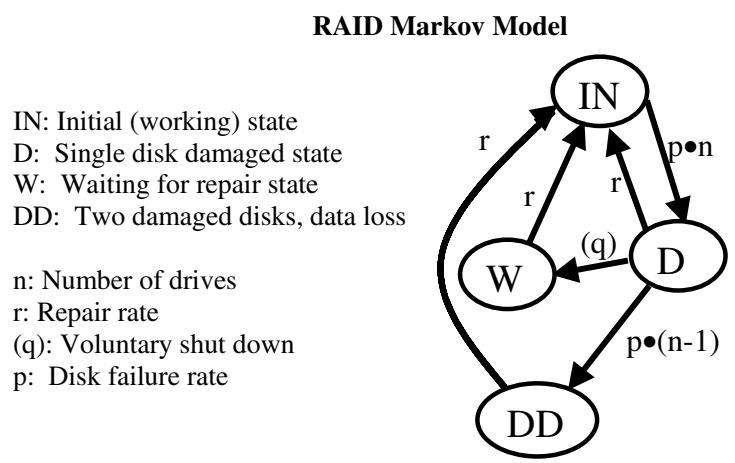

Fig. 13. Markov model for a RAID 5 system. from the uncertainties of the components and subsystems, each of which may be determined by a different person. Unlike the standard approach, fuzzy Markov modeling does not ignore these uncertainties; it preserves them, and provides an accurate mathematical evaluation of their combined effects.

\section{Fuzzy Markov modeling of a RAID system}

In an effort to demonstrate the wider applicability of fuzzy Markov modeling, we will now apply it to a RAID 5 system. The RAID (Redundant Array of Inexpensive Disks) [30] system has reliability characteristics quite similar to the MLDUA, despite considerable physical dissimilarity. RAID is a method to use multiple hard drives to increase the amount of data storage available, as often many smaller capacity drives are less expensive than the equivalent capacity single large drive. Additionally, most RAID systems also use one or more of the drives to increase reliability through redundancy. As RAID systems go through prototyping and design phases where failure rates are poorly known, fuzzy Markov modeling is a useful method of examining reliability issues.

The following example is of a RAID 5 system. In RAID 5 , coded information is spread throughout the RAID drives, so that if a drive is lost, all of the data stored on it can be retrieved from other disks [30,31]. This version is typical of the RAID approaches used in real systems, with $n+1$ drives storing $n$ drives worth of information to allow a single recoverable drive failure in any RAID group. The loss of two drives at the same time will result in lost data, however. Thus, a conservative system would shut down after a single drive failure, in hopes of preventing this. Conversely, a system designed to maximize data access would continue to operate, risking data loss. In this way, the reliability design for RAID 5 faces similar problems to those faced by the MLDUA operator.

Fig. 13 shows the Markov model used for the RAID system. Failure rates for the individual drives were fuzzified in the same way as the MLDUA components failure rates above from a base value of $2 \times 10^{-6}$ derived from Ref. [31]. Repair rates were also fuzzy: [0.004 0.020 .24 4] hours, covering expected repair times from four per hour to 10 days. The lower bound represents poor service or hard to find replacement drives, the upper bound the best possible replacement rate as mentioned in Ref. [31]. The one-cut values, representing $5-50 \mathrm{~h}$ repair times, represent institutional technical support levels. The voluntary shut down rate (q) was such that the system had 1 min after a transition to state $\mathrm{D}$ for another drive to fail before shut down (discretetime model) for the conservative system. This allows for the possibility of a transition to the lost data state in that time step, representing drive failures close enough together that the voluntary shut down routine has not finished yet.

Fig. 14 shows the fuzzy Markov model data for the 

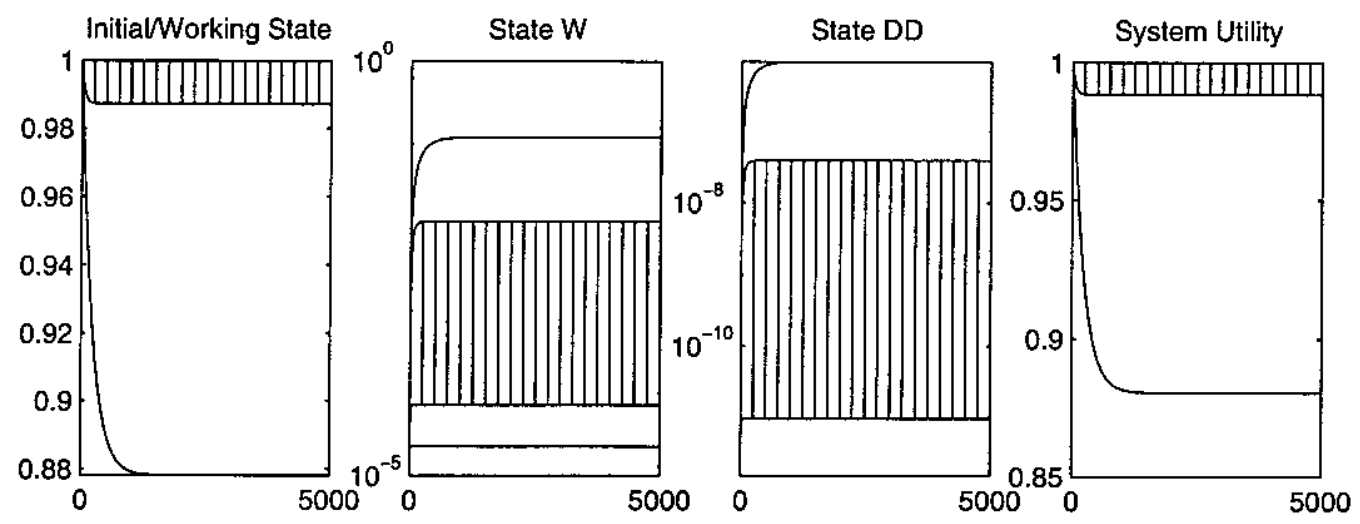

Fig. 14. RAID 5 Markov model output (population vs. hours) for a conservative operator.

conservative system. Note that data from state D is not shown, as the system voluntarily transitions out of this state immediately after entering it. The plot of the "system utility" represents the portion of the time the system may be expected to be operational, as it does with the MLDUA above. Since the conservative system shuts down after any disk failure it is possible that a significant population may develop for this state, corresponding to the real possibility of frequent loss of data access.

Fig. 15 shows the fuzzy Markov model data for the nonconservative system. Note that data from state $\mathrm{W}$ is not shown, as the system never enters it. For this model the system utility population is equal to one minus the population of state DD. The possible lower bound for utility is very close to one for this system, representing a smaller possibility of having an inoperative RAID drive, and thus a greater amount of working time. However, there is a much greater possibility of entering state DD, making actual data loss more likely. This is clearly seen if we compare the relative chances of data loss, as seen in Fig. 16, calculated using a model where the DD to IN repair transition has zero possibility, so data loss failures accumulate in state DD. In the worst case, the conservative operator is nearly 100 times less likely to lose any data.

Clearly, the RAID system shows similar behavior to the MLDUA with regards to the conservative versus non- conservative trade-off. This trade-off is most important for remote or hazardous environment systems that are unlikely to have easy access to data backups while simultaneously enduring conditions that subject its components to stress. It is also appropriate for prototyping and design phases where component characteristics and interaction may be poorly known. However, as discussed in Ref. [31] data storage requirements are growing quickly, and it is likely the reliability requirements of even office-bound RAID systems will become quite stringent, widening the applicability of the analysis.

\section{Conclusions}

The fuzzy Markov modeling approach introduced and discussed in this paper is a viable technique analyzing the reliability characteristics of complex systems. Previously existing fuzzy Markov approaches are not suitable for reliability analysis as discussed in this paper, although they are appropriate in the arenas in which they are used. The new method successfully propagates the fuzziness inherent in the data through the analysis without losing important information or becoming excessively complex. It works well in conjunction with fuzzy fault trees and system component grouping, allowing it to handle complex systems and benefit

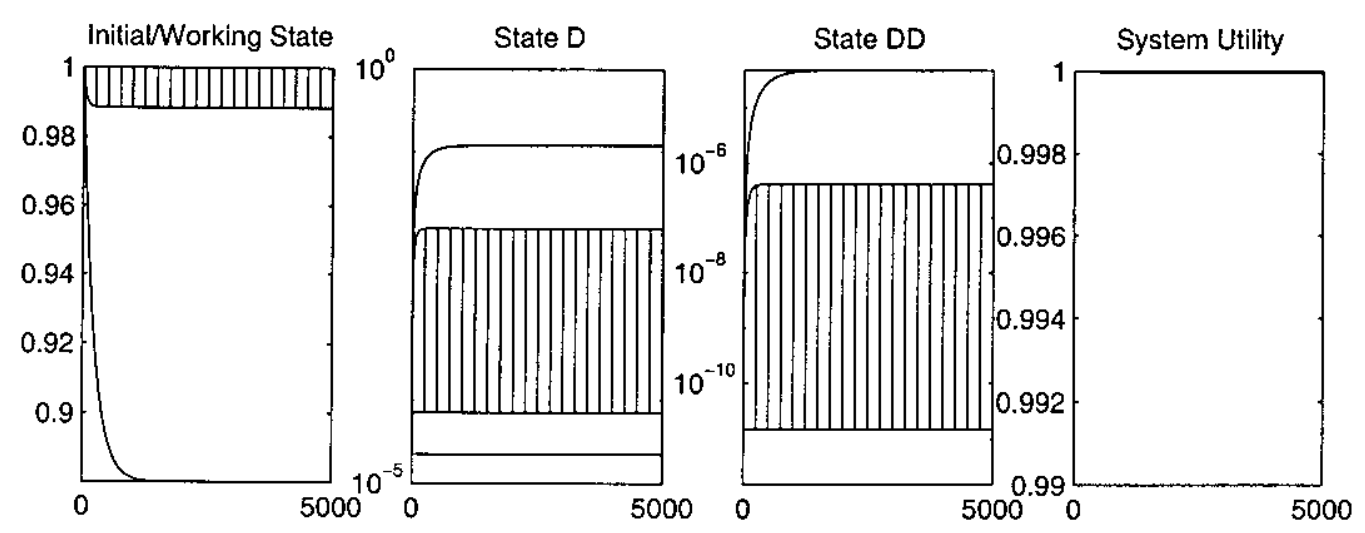

Fig. 15. RAID 5 Markov model output (population vs. hours) for a non-conservative operator. 


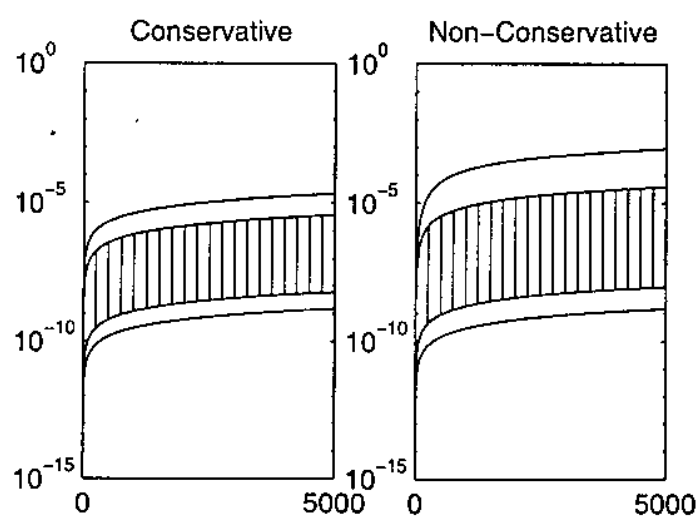

Fig. 16. RAID 5 Markov model: probability of data loss.

from existing reliability techniques and analyses. Although the calculations involved can be extensive, they are easily automated and the results are an intuitive fusion of existing fuzzy and reliability techniques.

The main drawback of the fuzzy Markov modeling method presented in this paper is its computational complexity. The complexity of the model increases exponentially with the number of fuzzy possibility distributions being considered. Currently, only simple or simplified models are solvable in a reasonable amount of time. However, the simplification of models using component grouping and fuzzy fault trees shows much promise as an integrated fuzzy reliability technique. Further reduction in computational complexity can be achieved by solving the Markov model symbolically rather than numerically, as the samples can then be substituted directly into the resulting equations, instead of re-solving the Markov model for every combination of samples.

Future work in the area of fuzzy Markov modeling is likely to focus on four areas. The first and most obvious of these is reduction of the computational complexity of the model. Similarly, further methods of simplification of the model should be considered, as the methods already considered lead to both useful solutions and better understanding of the systems being analyzed. Additionally, Markov modeling is a very broad area, and this work only considers fuzzification of the most basic of Markov models. Expanding this technique to some of the modified Markov models shows promise. The usefulness of the method introduced here as a part of a more far-reaching and complete reliability analysis involving fault detection and tolerance as seen in Refs. [3,8] and reconfiguration as seen in Ref. [5] is the future of current and ongoing work.

\section{Acknowledgements}

This work was supported in part by the National Science Foundation under grants IRI-9526363 and CMS-9796328, by DOE Sandia National Laboratory Contract \#AL3017, and DOE contract DE-FG07-97ER 14830.

\section{References}

[1] English JD, Maciejewski AA. Euclidean-space measures of robotic joint failures. In: IEEE International Conference on Robotics and Automation, Albuquerque, NM, 1997. p. 2894-901.

[2] Paredis CJJ, Khosla PK. Mapping tasks into fault tolerant manipulators. In: Proceedings of the IEEE International Conference on Robotics and Automation, San Diego, CA, vol. 1, 1994. p. 696-703.

[3] Visinsky ML, Cavallaro JR, Walker ID. A dynamic fault tolerance framework for remote robots. IEEE Trans Robotics Automation 1995;11(4):477-90.

[4] Lewis CL, Maciejewski AA. An example of failure tolerant operation of a kinematically redundant manipulator. In: Proceedings of the IEEE International Conference on Robotics and Automation, San Diego, CA, vol. 2, 1994. p. 1380-7.

[5] Lewis CL, Maciejewski AA. Dexterity optimization of kinematically redundant manipulators in the presence of joint failures. Int J Comput Electrical Engng 1994;20(3):273-88.

[6] Paredis CJJ, Au WKF, Khosla PK. Kinematic design of fault tolerant manipulators. Int J Comput Electrical Engng 1994;20(3):211-20.

[7] Ting Y, Tosunoglu S, Tesar D. A control structure for fault tolerant operation of robotic manipulators. In: Proceedings of the IEEE International Conference on Robotics and Automation, Atlanta, GA, 1993. p. 684-90.

[8] Visinsky ML, Cavallaro JR, Walker ID. Robotic fault detection and fault tolerance: a survey. Reliab Engng System Safety 1994;46(2):139-58.

[9] Cavallaro JR, Walker ID. Failure mode analysis of a proposed manipulator-based hazardous material retrieval system. In: ANS Seventh Topical Meeting on Robotics and Remote Systems, Augusta, GA, vol. 2, 1997. p. 1096-102.

[10] Walker ID, Cavallaro JR. The use of fault trees for the design of robots for hazardous environments. In: Proceedings of the IEEE Annual Reliability and Maintainability Symposium, Las Vegas, NV, 1996. p. 229-35.

[11] Lin C, Wang MJ. Hybrid fault tree analysis using fuzzy sets. Reliab Engng System Safety 1997;58(3):205-13.

[12] Dhillon BS. Robot reliability and safety. New York: Springer, 1991.

[13] Leuschen ML. Robot reliability through fuzzy Markov models. Masters thesis, ECE Department, Rice University, Houston, TX, 1997.

[14] Leuschen ML, Cavallaro JR, Walker ID. Robot reliability through fuzzy Markov models. In: Proceedings of the IEEE Annual Reliability and Maintainability Symposium, Anaheim, CA, 1998. p. 209-14.

[15] Cai KY. System failure engineering and fuzzy methodology: an introductory overview. Fuzzy Sets and Systems 1995;83(2):113-33.

[16] Onisawa T, Nishiwaki Y. Fuzzy human reliability analysis on the Chernobyl accident. Fuzzy Sets and Systems 1988;28(2):115-27.

[17] Denson W, Chandler G, Crowell W, Clark A, Jaworski P. Nonelectric parts reliability data. Technical Report NPRD-95, Reliability Analysis Center, Rome, NY, 1994.

[18] Kozine IO, Filimonov YV. Imprecise reliabilities: experiences and advances. Reliab Engng System Safety 2000;67:75-83.

[19] Bechta-Dugan J, Venkataraman B, Gulati R. DIFtree: a software package for the analysis of dynamic fault tree models. In: Proceedings of the IEEE Annual Reliability and Maintainability Symposium, Philadelphia, PA, 1997. p. 64-70.

[20] Harpel BM, Bechta-Dugan J, Cavallaro JR, Walker ID. Analysis of robots for hazardous environments. In: Proceedings of the IEEE Annual Reliability and Maintainability Symposium, Philadelphia, PA, 1997. p. 111-6.

[21] Tanaka H, Fan LT, Lai FS, Toguchi K. Fault tree analysis for fuzzy probability. IEEE Trans Reliab 1983;R-32(5):453-7.

[22] de Campos LM, Bolanos MJ. Characterization and comparison of Sugeno and Choquet integrals. Fuzzy Sets and Systems 1992;52(1):61-7. 
[23] Mohamed M, Gader P. Generalization of hidden Markov models using fuzzy integrals. In: Proceedings of the First International Joint Conference of NAFIPS/IFIS/NASA, San Antonio, TX, 1994. p. 3-7.

[24] Leuschen ML, Walker ID, Cavallaro JR. Robot reliability using fuzzy fault trees and Markov models. In: SPIE Proceedings on Sensor Fusion and Distributed Robotic Agents, Boston, MA, vol. 2905, 1996. p. 73-91.

[25] Mares M. Computation over fuzzy quantities. Boca Raton, FL: CRC Press, 1994.

[26] Moore RE. Methods and applications of interval analysis. Philadelphia, PA: SIAM Press, 1979.

[27] Cooper JA. Constrained mathematics evaluation in probabilistic logic analysis. Reliab Engng System Safety 1998;60:199-203.
[28] Bechta-Dugan J, Bavuso SJ, Boyd MA. Dynamic fault-tree models for fault-tolerant computer systems. IEEE Trans Reliab 1992;41:36377.

[29] Love L, Kress R, Bills K. Simulation tools for robotic and teleoperated hazardous waste removal. In: Proceedings of the IEEE International Conference on Robotics and Automation, Albuquerque, NM, 1997. p. 66-71.

[30] Stallings W. Computer organization and architecture. Upper Saddle River, NJ: Prentice-Hall, 1999.

[31] Burkhard WA, Menon J. Disk array storage system reliability. In: Proceedings of the 23rd International Symposium on Fault-Tolerant Computing, Toulouse, France, 1993. p. 432-41. 